\title{
Electrostatic Force Microscopy Characterization of Trioctylphosphine Oxide Self-assembled Monolayers on Graphite
}

\author{
Jiang Jiang, Todd D. Krauss, and Louis E. Brus* \\ Department of Chemsitry, Columbia University, New York, New York 10027
}

Received: May 2, 2000

\begin{abstract}
We have studied the self-assembling behavior of trioctylphosphine oxide (TOPO) on a highly oriented pyrolytic graphite surface. TOPO forms stripe-like structures in registry with the underlying graphite lattice. Using electrostatic force microscopy, we have also measured the charge, dipole, and dielectric constant of these monolayer films. The crystalline stripe phase has a net positive charge of about $2 \times 10^{-5}$ electron charge per TOPO molecule. The surface dipole due to adsorption is extremely small; this result implies that the dipole moment of TOPO is oriented parallel to the surface. Perfect image charges are not formed inside the graphite.
\end{abstract}

\section{Introduction}

Molecular thin films are important for basic science research as well as for technological applications. ${ }^{1}$ Such films provide a flexible and easy way to modify surfaces to enable the systematic study of surface and interfacial properties. Molecular thin films can also be used for optical devices, liquid crystal displays, chemical and biological sensors, and molecular switches. ${ }^{2}$ Despite all the excitement and promise of this field, our understanding of molecular thin films is still limited.

Self-assembled monolayers $(\mathrm{SAMs})^{3}$ are ordered molecular assemblies formed by spontaneous adsorption of molecules onto a solid support surface. Compared to traditional lithographic techniques, self-assembly is potentially a much easier and more economical way to build 2D or even 3D structures. Our current understanding of SAMs results mostly from studies of linear or planar molecules. Among them, SAMs of alkanethiols on gold have been the subject of intensive study. ${ }^{4}$ However, little attention has been paid to self-organization of nonplanar molecules. ${ }^{5}$

Trioctylphosphine oxide (TOPO) contains three hydrocarbon chains resulting in local trigonal symmetry, and also has a large permanent dipole moment from the phosphorus-oxygen bond. It has been widely used as a monodentate uncharged ligand for metal ion extraction, ${ }^{6}$ and also as an auxiliary ligand to improve the extractability of coordinatively unsaturated metal complexes. ${ }^{7}$ It acts as both the solvent and the capping molecule in the synthesis of monodispersed semiconductor nanocrystals, ${ }^{8}$ which have great importance in scientific study and applications. ${ }^{9,10}$

Characterization of SAMs by macroscopic techniques, such as ellipsometry and contact angle studies, can determine physical properties of SAMs, such as film thickness, on the millimeter scale. ${ }^{4}$ Various diffraction techniques ${ }^{11}$ have been used to determine the lattice constants and molecular orientation of SAMs. The invention of the scanning probe microscope $(\mathrm{SPM})^{12,13}$ has provided scientists a powerful nondestructive probe for the study of thin films on the molecular and atomic level. Studies of the lattice structure, ${ }^{14}$ and self-assembly mechanism ${ }^{15}$ of alkanethiol SAMs have been achieved with atomic resolution by SPM. Electrostatic force microscopy

* Author to whom correspondence should be addressed.
(EFM), ${ }^{16}$ a variant of atomic force microscopy (AFM) ${ }^{13}$ can be used to measure the electrostatic force between a conductive AFM tip and a sample surface. Specifically, EFM can measure dielectric properties, ${ }^{16}$ surface charges, ${ }^{17-20}$ as well as contact potential differences ${ }^{21,22}$ with nanometer resolution. EFM also provides a noninvasive surface potential measurement for semiconductor devices ${ }^{23}$ and ferroelectric materials, ${ }^{24}$ and it has the potential to study biological species. ${ }^{25}$

Here, we present studies of TOPO molecular self-assembly on an atomic flat highly oriented pyrolytic graphite (HOPG) surface. We found that TOPO forms stripe-like monolayer structures in registry with the graphite lattice, which are positively charged. We infer from our results that HOPG does not screen the TOPO dipole as expected for a perfect metal.

\section{Theory}

An EFM measurement is based on the long-range electrostatic interaction between a conductive AFM tip and a metal surface. To calculate the electrostatic forces acting on the EFM tip, we treat the tip-substrate force as a capacitive interaction, and the force between the tip and any surface charges as due to Coulombic interactions. ${ }^{18}$ By applying an external bias voltage $V=V_{\mathrm{dc}}+V_{\mathrm{ac}} \sin (\omega t)$ to the tip and the conductive substrate, the tip experiences electrostatic forces at zero frequency, $\omega$, and $2 \omega$. We can write out the components of the force acting on the tip at $\omega$ and $2 \omega$ as

$$
F_{\text {tip }}(\omega)=\left(V_{\mathrm{dc}}+\phi\right) V_{\mathrm{ac}} \frac{\partial C}{\partial z}+E_{z} Q_{\text {tip }}
$$

and

$$
F_{\text {tip }}(2 \omega)=\frac{1 \partial C}{4 \partial z} V_{\mathrm{ac}}^{2}
$$

In eqs 1 and $2, C$ is the tip-substrate capacitance, $z$ is the tipto-substrate distance, and $\phi$ is the contact potential difference between the tip and the substrate. For clean surfaces in a vacuum, $\phi$ is given by $\left(W_{\text {substrate }}-W_{\text {tip }}\right) /(-e)$, where $e$ is the electron charge. $W_{\text {substrate }}$ and $W_{\text {tip }}$ are the work functions of the substrate and the tip, respectively. $Q_{\text {tip }}$ is the charge on the EFM tip, which is equal to $C\left(V_{\mathrm{dc}}+\phi+V_{\mathrm{ac}} \sin (\omega t)\right)$ plus the induced charge due to any localized surface charges inside the capacitor. 
$E_{z}$ is the $z$ component of the electric field above the surface. By adjusting the $V_{\mathrm{dc}}$ to null out $\phi$, this technique can provide a direct measure of $\phi$ in the absence of surface charges. In the presence of surface charge, we can adjust $V_{\mathrm{dc}}$ to zero the first term in eq 1 , and thus determine the magnitude and the sign of the charge on the surface with nanometer spatial resolution. ${ }^{18}$ From the force on the tip at $2 \omega$, we can obtain the information about local dielectric properties through the $\mathrm{d} C / \mathrm{d} z$ term. ${ }^{16}$

As an EFM tip is scanned above a surface, small force gradient changes above the surface will modify the effective spring constant of the vibrating cantilever $(k)$, thus affecting its resonant frequency $(v)$. The relationship between the cantilever frequency change $(\Delta v)$ and the force gradient in the $z$ direction is given by $^{26}$

$$
v^{\prime}=v \sqrt{1-\frac{1 \partial F}{k \partial z}}
$$

For $\Delta v=v^{\prime}-v \ll v$, the force gradient can be approximated as

$$
\frac{\partial F}{\partial z}=-2 k \frac{\Delta v}{v}
$$

If the surface is uncharged, $E_{z}=0$, and this enables a measurement of the capacitance of the tip-substrate system. The capacitance is obtained from $\mathrm{d}^{2} \mathrm{C} / \mathrm{d} z^{2}$, which is given by

$$
\frac{\partial^{2} C}{\partial z^{2}}=-\frac{2 k \quad \Delta v(\omega)}{\left(V_{\mathrm{dc}}+\phi\right) V_{\mathrm{ac}} \quad v}
$$

By measuring $\Delta v(\omega)$ as a function of $z$ for fixed $V_{\text {ac }}$ and $V_{\mathrm{dc}}$, we can obtain the tip-substrate capacitance by integrating over eq 5 twice.

A finite-sized monolayer of TOPO with uniform orientation might be modeled as a dipole layer. For a rectangular dipole layer centered at the origin with dimensions of $L$ by $M$, the electric potential at an arbitrary point $(x, y, z)$ can be written as

$$
\begin{aligned}
& V(x, y, z)= \\
& \quad \int_{-L / 2}^{L / 2} \int_{-M / 2}^{M / 2} \frac{p}{4 \pi \epsilon_{0}\left[\left(x_{1}-x\right)^{2}+\left(y_{1}-y\right)^{2}+z^{2}\right]^{3 / 2}} \mathrm{~d} x_{1} \mathrm{~d} y_{1}
\end{aligned}
$$

In the above equation, $p$ is the dipole moment of an individual molecule, and $\epsilon_{0}$ is the permittivity of the free space. The $z$ component of the electric field of a TOPO layer can be obtained by taking the gradient of the electric potential $E_{z}=-\mathrm{d} V / \mathrm{d} z$.

A molecular or physical dipole can be modeled as a sheet of negative charges and a sheet of positive charges separated by a distance $d .^{27}$ The charge density $\sigma$ can be expressed as $\sigma=$ $p A / d$, where $A$ (molecule $/ \mathrm{nm}^{2}$ ) represents the molecular packing density on the surface. For this case, the electric field normal to the surface can be expressed as

$$
\begin{aligned}
& E_{z}(x, y, z)= \\
& \int_{-L / 2}^{L / 2} \int_{-M / 2}^{M / 2} \frac{\sigma}{4 \pi \epsilon_{0}}\left\{\frac{z}{\left[\left(x_{1}-x\right)^{2}+\left(\mathrm{y}_{1}-\mathrm{y}\right)^{2}+\mathrm{z}^{2}\right]^{3 / 2}}-\right. \\
& \\
& \left.\frac{z}{\left[\left(x_{1}-x\right)^{2}+\left(y_{1}-y\right)^{2}+(z+d)^{2}\right]^{3 / 2}}\right\} \mathrm{d} x_{1} \mathrm{~d} y_{1}
\end{aligned}
$$

The electric field obtained from eqs 6 and 7 is only correct for a dipole layer in free space. In the presence of a metal or a dielectric substrate, the electric field above the surface can be expressed as the superposition of the field from real charges,
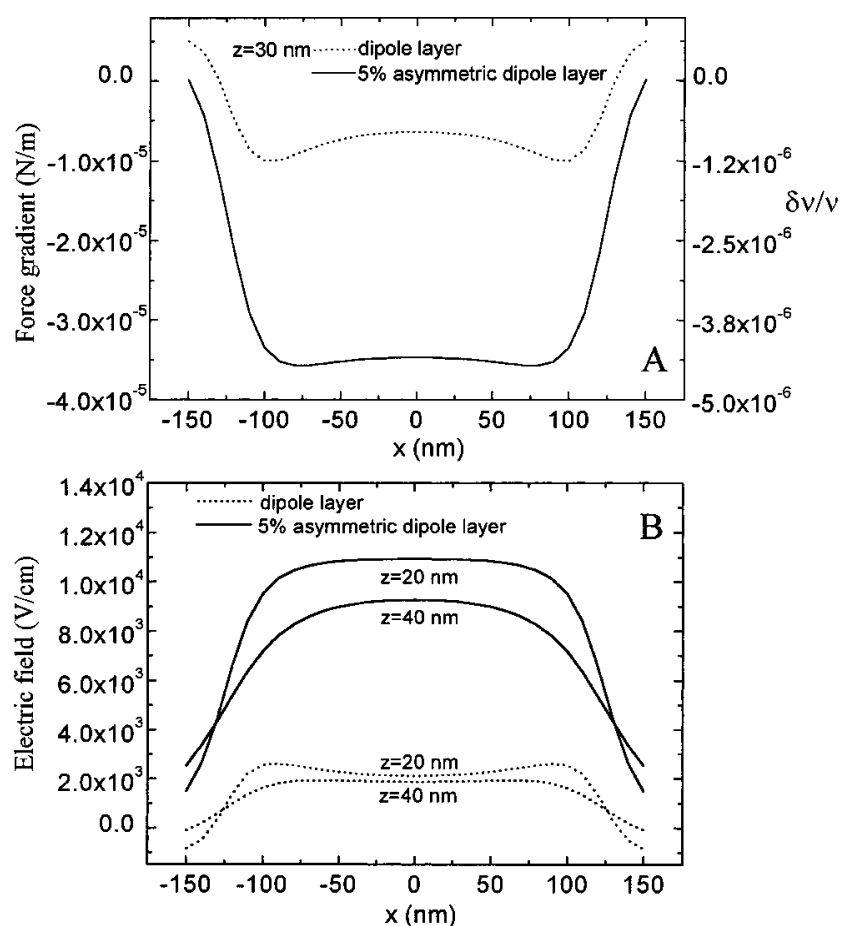

Figure 1. Calculated force gradient (A) and electric field (B) over a $250 \mathrm{~nm} \times 250 \mathrm{~nm}$ TOPO patch for a perfect dipole layer, and an asymmetric dipole layer with $5 \%$ uncompensation. Charge density is $2 \times 10^{-3} \mathrm{e} / \mathrm{nm}^{2}$.

and the field from image charges inside the substrate. From classical electrostatic theory, the electric field due to a point charge $q$ outside the surface of a perfect conductor contains an additional contribution from that of a charge $-q$ at its image point with respect to the surface. Thus, the real molecular dipole will have a corresponding image dipole. The resulting electric field will be a superposition of two dipole fields, one from the real dipole, and one from the image dipole. If one charged sheet is directly on the surface, the charge and its corresponding image charge would be very close to each other. Thus, we approximate the physical dipole and its image as positive and negative charge planes separated by $2 d$.

To account for possible charge transfer between HOPG and TOPO, and the possible poor image charge formation inside HOPG as indicated by its nonideal metallic behavior, ${ }^{28,29}$ an asymmetric dipole layer is also modeled. The asymmetric dipole layer consists of a charge plane and an image plane with nonequal charge density, which is equivalent to the superposition of a charge plane and a dipole sheet.

The calculated electric field and electrostatic force gradient for both a perfect dipole layer and an asymmetric dipole layer with a 5\% charge uncompensation are shown in Figure 1. The percentage of uncompensation is defined as the ratio of the difference between the magnitude of the partial image charge and the perfect image charge, and the magnitude of the partial image charge. The curves shown are crosscuts along one of the axes of a square patch of size $250 \mathrm{~nm}$ by $250 \mathrm{~nm}$. For a perfect dipole layer, there is a fringing effect at the edges of the dipole patch. The electric field and force gradient reverse sign crossing the edge, and the force gradient will have its maximum along the edge. However, the electric field and the force gradient are fairly uniform across an asymmetric dipole layer. Thus, it behaves more like a charge plane, which has a uniform electric field and force gradient, than a dipole sheet. Also, the electric field and the force gradient for a 5\% asymmetrical dipole layer will be 4 or 5 times larger than that for a perfect dipole layer 


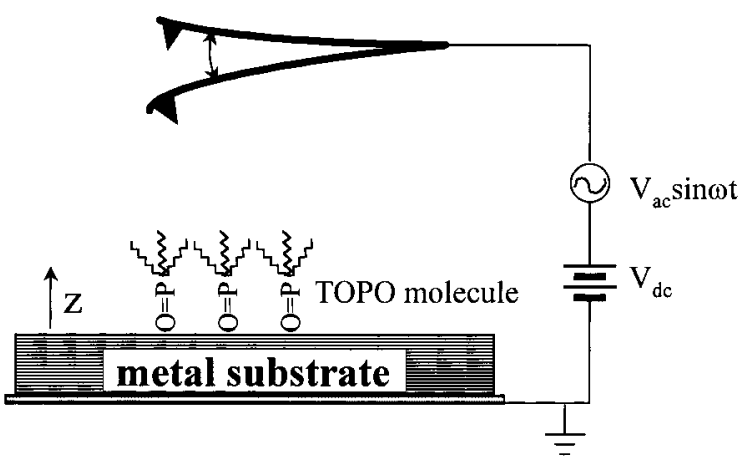

Figure 2. Schematic diagram of EFM experimental setup.

for equal charge densities. Thus, the electric field is dominated by small amounts of charge, even in the presence of a dipole layer.

\section{Experimental Section}

TOPO layers on HOPG were prepared by liquid deposition. TOPO $(99 \%)$ and anhydrous hexane $(95+\%)$ were purchased from Aldrich. To prepare a sample, one drop of TOPO hexane solution (typically less than $10^{-9} \mathrm{M}$ ) was deposited onto the basal plane of freshly cleaved HOPG. ${ }^{30}$ We took AFM and EFM images of the sample using a Nanoscope IIIa MultiMode AFM from Digital Instruments. The tips used were commercial uncoated and cobalt-chromium-coated $\mathrm{Si}$ cantilevers ${ }^{31}$ with spring constants ranging from 1 to $5 \mathrm{~N} / \mathrm{m}$. Gold substrates were prepared by evaporating $500 \mathrm{~nm}$ thick Au onto heated mica.

To map the surface topography and electrostatic properties simultaneously and independently, we used interleave scanning and linear lift mode, features of the MultiMode AFM. Each scan in lift mode consists of two passes. The first pass was a tapping mode AFM scan with no external voltage applied, obtaining the surface topography. We operated the tip at its resonance frequency $(60-90 \mathrm{kHz})$. Next, the tip was lifted up at a certain height above the surface and scanned at constant height during the second pass while a variable dc and ac voltage was applied to the tip. Lock-in amplifiers were used to measure the cantilever frequency shift at $\omega$ and $2 \omega$ simultaneously. A schematic graph of the EFM setup is shown in Figure 2. All EFM measurements were carried out at room temperature inside a glovebox with a relative humidity of less than $3 \%$ to eliminate possible screening effects of a water film on the sample surface.

Typical parameters for EFM measurements were the following: ac voltage $V_{\text {ac }}$ was $6 \mathrm{~V}$ peak-to-peak, $\omega$ was $2 \pi \times 800$ $\mathrm{Hz}$, lock-in time constant was $3 \mathrm{~ms}$, image scan rate was 1.25 $\mathrm{Hz}$, tip-to-sample separation was $\sim 30 \mathrm{~nm}$, and the acquisition time for a $256 \times 256$ pixel EFM image was 6-7 min.

\section{Results}

The self-assembly of TOPO on HOPG shows stripe-like structures at very low TOPO concentrations $\left(\sim 10^{-11} \mathrm{M}\right)$. An AFM height image of these structures is shown in Figure 3A. From the cross-section profile, we measure the height of these stripe structures to be $\sim 0.7 \mathrm{~nm}$, which is the approximate length of one TOPO molecule. The height is very uniform both along the stripe and among stripes. The typical width ranges from 70 to $150 \mathrm{~nm}$, and the length can be up to micrometers. A striking feature of these stripe structures is the $60^{\circ}$ or $120^{\circ}$ angle formed between the stripes.

The striped structures are not thermodynamically stable, and will evolve with time and eventually form large aggregates with a characteristic size of micrometers. This suggests that TOPO

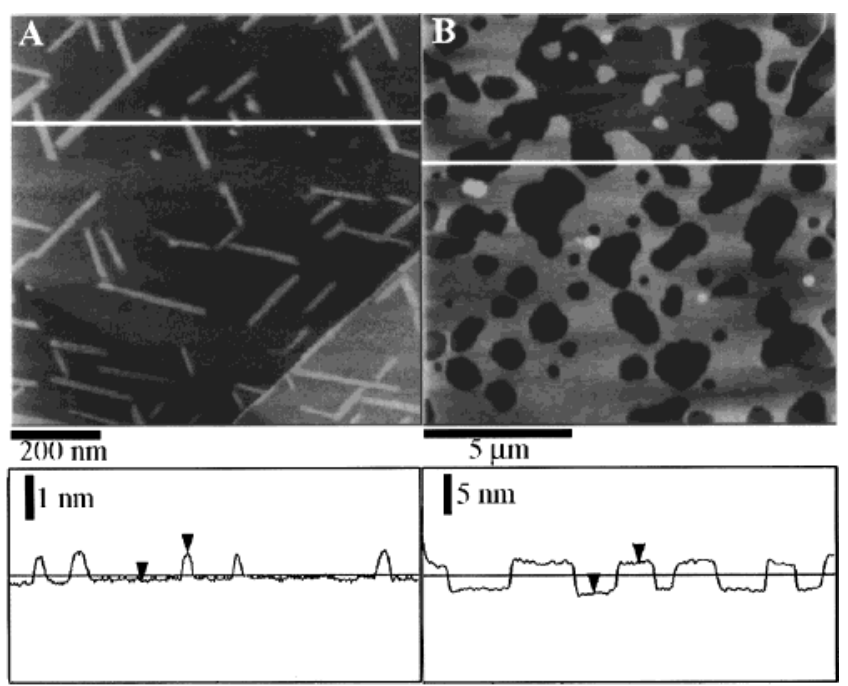

Figure 3. AFM height image and the cross section profile for TOPO on HOPG at concentrations (A) $10^{-11} \mathrm{M}$ and (B) $10^{-9} \mathrm{M}$. The holes in (B) are bare HOPG.

molecules have higher intermolecular interactions with each other than with the graphite surface. This view is supported by studies that TOPO will form dimers in solution because of strong intermolecular interactions. ${ }^{32}$

At a higher concentration $\left(\sim 10^{-9} \mathrm{M}\right)$, TOPO molecules will form multilayered structures on HOPG. Depending on the local concentration and the drying process, TOPO molecules will form either a multilayered network or multilayered islands. An example of a multilayered network coexisting with bare HOPG is shown in Figure 3B. Adsorption of TOPO on top of an existing monolayer occurs preferentially. This is further evidence that TOPO intermolecular interactions are stronger than that between TOPO and HOPG. We have not been able to obtain a well-formed nonstripe monolayer of TOPO by the liquid deposition method. Multilayer stripe structures are also not observed.

The $z$ dependence of the tip-substrate capacitance is an important parameter, and is necessary for the calculation of surface charge and local dielectric constant. Instead of calculating $C(z)$ assuming a certain tip geometry, we can directly measure the capacitance derivative by measuring $\Delta v(\omega)$ as a function of $z$ for a freshly peeled HOPG, and determine $C(z)$ using eq 5 . We find that the second derivative of the capacitance has a $z$ dependence $\mathrm{d}^{2} C / \mathrm{d} z^{2} \sim z^{-1.5}$ which is between a sphereplane $\left(\mathrm{d}^{2} C / \mathrm{d} z^{2} \sim z^{-2}\right)$ and cone-plane geometry $\left(\mathrm{d}^{2} C / \mathrm{d} z^{2} \sim\right.$ $\left.z^{-1}\right){ }^{19,33}$ This seems like a reasonable value for $\mathrm{d}^{2} C / \mathrm{d} z^{2}$ for a square-pyramidal-shaped EFM tip, which has a geometry between a cone and a sphere.

Using EFM, we have measured the contact potential differences between an EFM tip and Au, and an EFM tip and HOPG. $\phi$ is plotted in Figure 4 as a function of the tip-substrate separation. For $\mathrm{Au}$, in the $z$ range that we have tested, $\phi$ is nearly constant, as expected from simple theory. For HOPG, $\phi$ decreases as $z$ is increased.

An EFM image of a submonolayer of TOPO on HOPG is shown in Figure 5. Figure 5B shows the topography of the assembled TOPO on HOPG. Figures $5 \mathrm{~A}$ and $5 \mathrm{C}$ show the change in resonant frequency $(\Delta v)$ at $\omega$ and $2 \omega$, respectively. The TOPO is dark with respect to HOPG in Figure 5A. This corresponds to TOPO having a positive charge, or a dipole with the positive end up. Interestingly, we observed alternating positive charge and negative charge signals for different layers of the multilayered structure. 


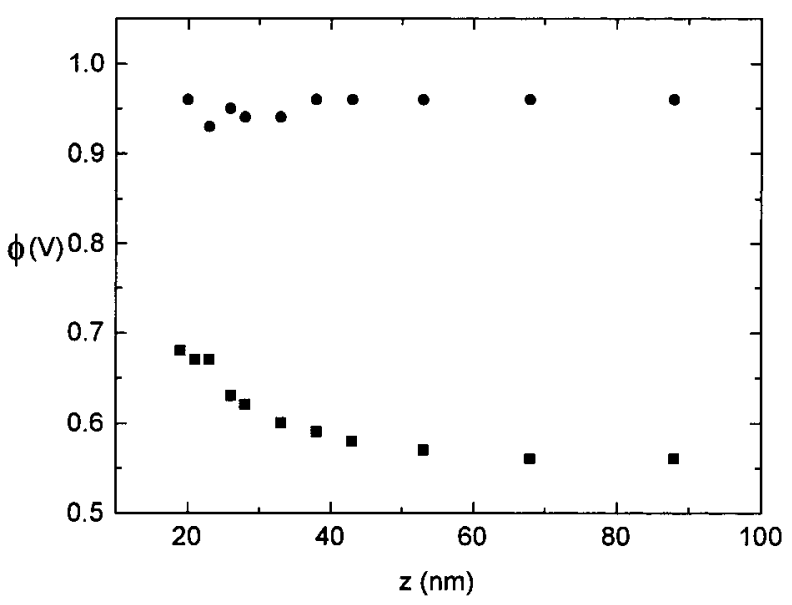

Figure 4. Tip-substrate contact potential difference as a function of the tip-substrate separation for Au (dots) and HOPG (squares). The amplitude setpoint is $0.4 \mathrm{~V}$ which corresponds to a peak-to-peak amplitude of $16 \mathrm{~nm}$ for full oscillation of cantilever on the surface. The average height of the tip starts from $19 \mathrm{~nm}$.

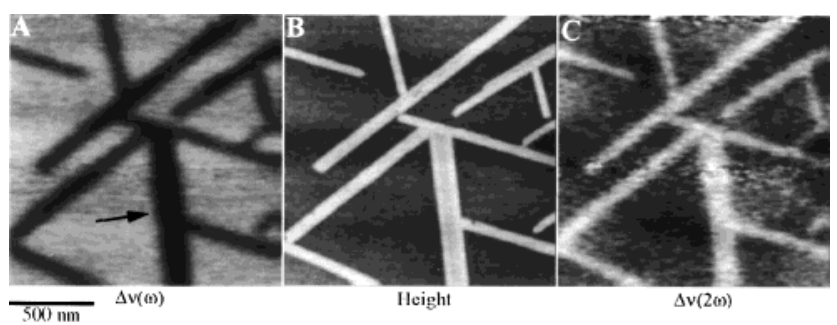

Figure 5. EFM image of TOPO on HOPG with $V_{\mathrm{dc}}+\phi=0$. The amplitude setpoint is $0.7 \mathrm{~V}$ corresponding to a full amplitude of 28 $\mathrm{nm}$. The lift height is $15 \mathrm{~nm}$.

Comparing the measured force gradient over a TOPO stripe with calculated values, we can assess whether $\Delta v(\omega)$ is due solely to a dipole field. Experimental results show the force gradient over a TOPO patch is quite uniform. For a perfect dipole layer, one expects the force gradient to have a maximum near the edge, and a dip in the center. Also, just outside the TOPO layer, the dipole force gradient should reverse sign. Thus, we find that a pure dipole field does not fit well with the experimental cross section profile. However, an asymmetric dipole layer with a certain uncompensation percentage $(\geq 5 \%)$ does fit the data shape, but not the magnitude, as shown in Figure 6. At this level of uncompensation, the fit only gives an upper limit for the dipole contribution on top of the uncompensated positive charge contribution.

We can calculate the charge density magnitude from $\Delta v(\omega)$ for a given TOPO stripe. We found that $\Delta v(\omega)$ goes to zero over a TOPO stripe when $V_{\mathrm{dc}}+\phi \sim 0.02 \mathrm{~V}$. Using eqs 1 and 4 , this allows us to calculate the absolute magnitude of $E_{z}$ above the TOPO stripe. With an asymmetric dipole layer with a 5\% or more uncompensation, the calculated charge density of the dipole layer has an upper limit of $4 \times 10^{-4} \mathrm{e} / \mathrm{nm}^{2}$, and the uncompensated positive charge density is $2 \times 10^{-5} \mathrm{e} / \mathrm{nm}^{2}$. As the area of one TOPO molecule is $\sim 1 \mathrm{~nm}^{2}$, the net charge per TOPO in the stripe is $\sim 2 \times 10^{-5}$ electron charge. If we assume the measured $E_{z}$ is due solely to charge transfer from TOPO to HOPG, then this charge transfer is partially screened. For 5\% imperfect screening, the actual electron transfer is $\sim 4 \times 10^{-4}$ $e / \mathrm{nm}^{2}$. This number is an upper limit. Thus, the actual charge transfer is low, consistent with van der Waals bonding.

The magnitude of the force gradient at $2 \omega$ depends on the dielectric constant of the TOPO molecule through the $\mathrm{d} C / \mathrm{d} z$ term in eq 2. Also, $\Delta v(2 \omega)$ depends linearly on the cantilever spring constant through eq 4 . By fitting $\Delta v(2 \omega)$ over a TOPO stripe, we infer the dielectric constant $\epsilon$ of TOPO to be $\sim 2.5$. This result is close to the literature value of $2.6 .^{34}$

\section{Adsorption, Dipole Moment, and Screening}

TOPO stripes are in registry with the underlying graphite lattice, which consists of a hexagonal honeycomb structure formed by carbon atoms. By occupying one of the three equivalent adsorption sites, ${ }^{35}$ TOPO stripes can be viewed as hexagonal domains. Thus, TOPO domains will have a $60^{\circ}$ or $120^{\circ}$ angle between them. The registry of these stripe structures with the graphite lattice indicates that the underlying graphite directs TOPO layer formation at very low concentrations.

The dominant EFM signal results from uncompensated positive charge. As previously discussed, this could reflect charge transfer from TOPO to graphite, coupled with the absence of a complete image charge in the graphite. It might also reflect image charge formation of different magnitude for the positive and negative parts of the TOPO dipole.

For TOPO multilayers, the observed $\Delta v(\omega)$ originates from the dipole moment of the TOPO molecule because the sign of the signal reverses for different multilayer heights. This can be qualitatively understood by arguing that the TOPO multilayers are stacked in a head-to-head and tail-to-tail configuration, and perpendicular to the surface. Quantitative understanding is difficult due to uncertainty in the exact number of TOPO layers.

For TOPO stripes, the upper limit of the calculated dipole density is about 170 times lower than expected for a dense layer of TOPO with the $C_{3 v}$ axis normal to the surface. ${ }^{36,37}$ The calculated value for the molecular packing density of TOPO is based on the assumption that the dipole moment of TOPO is normal to the surface. Since EFM only measures the force gradient normal to the surface, a dipole normal to the surface will have a larger signal than that of a tilted dipole. The EFM signal at $\omega$ will disappear completely if the dipole is parallel to the surface. Thus, for the same magnitude signal, the packing density of tilted dipoles must be higher than that of dipoles normal to the surface. Therefore, one reason the calculated charge density is so low is that TOPO may be tilted on the surface, having the $C_{3 v}$ axis at an angle to the surface normal.

Studies of long hydrocarbon chain molecules physisorbed on a graphite surface ${ }^{38}$ provide evidence that TOPO does not assemble on HOPG with the dipole normal to the surface. Long hydrocarbon chain molecules tend to lie down on the surface forming a commensurate structure. ${ }^{38}$ This is due to two factors: ${ }^{39}$ (1) strong van der Waals interaction between the hydrocarbons and the graphite; and (2) the matching between the hydrocarbon backbones and the graphite lattice.

Studies of polar pyramidal molecules of methane derivatives physisorbed on graphite ${ }^{40}$ provide further information as to the nature of the assembly of TOPO on HOPG. These studies found that van der Waals interactions will bring as many atoms to the surface as possible, and electrostatic interactions will bring the charge centers as close to the surface as possible. The net result is a tripod geometry, which has two stable orientations on the surface. ${ }^{40}$ A local energy minimum exists when $\theta$, the angle between the dipole moment and the surface normal plane, is equal to zero. The global minimum orientation has $\theta \sim 90^{\circ}$. By putting the two of the hydrocarbon chains of TOPO on the surface, the van der Waals interaction is maximized. Furthermore, by putting the polar head near the surface at a very large angle from the surface normal, electrostatic energy is minimized. In addition, by adapting this configuration, the TOPO molecules 

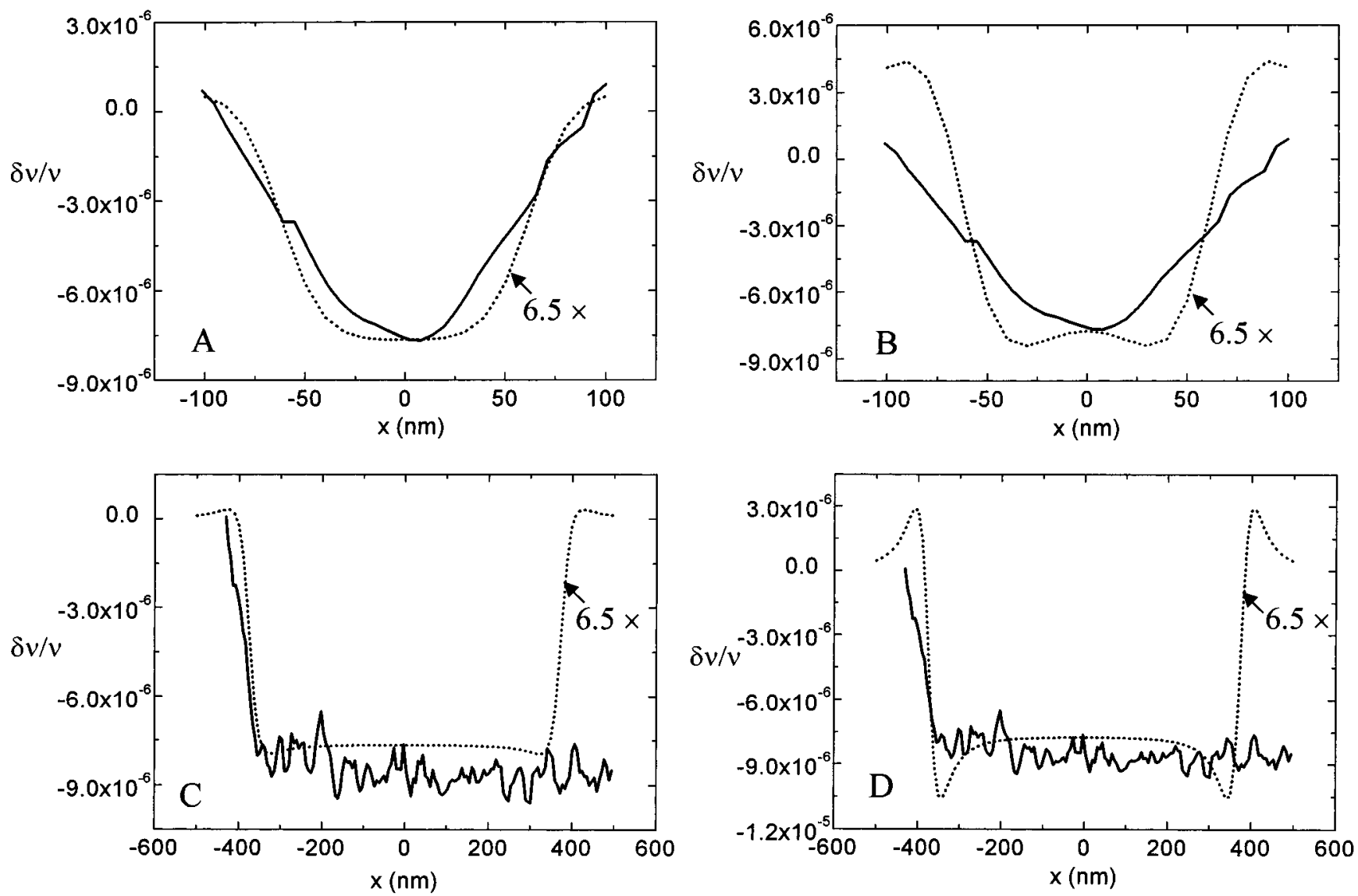

Figure 6. Comparison of the experimental (solid curve) and calculated (dotted curve) force gradient over the TOPO stripe indicated in Figure 5, both along the short axis(A),(B) and the long axis(C),(D). The calculation is based on a $125 \mathrm{~nm} \times 750 \mathrm{~nm}$ TOPO patch for a $5 \%$ asymmetric dipole layer (A),(C) with charge density of $4 \times 10^{-4} \mathrm{e} / \mathrm{nm}^{2}$ and for a perfect dipole layer (B),(D) with charge density of $2 \times 10^{-3} \mathrm{e} / \mathrm{nm}^{2}$. The tip-sample distance is $30 \mathrm{~nm}$. The calculated results are multiplied by a factor of 6.5 .

could form an antiferroelectric layer which favors the intermolecular dipole-dipole attraction.

HOPG has shown anomalous behavior compared to other metals. For example, the surface dielectric response of graphite is very different from a free-electron metal due to its semimetallic band structure. ${ }^{28}$ Also, instead of being quenched, photoluminescence of semiconductor nanocrystals was observed on a graphite surface. ${ }^{29}$ Thus, treating graphite as a high-density, free-electron metal in the jellium approximation, and ignoring its band structure near Fermi level, seems not to be accurate.

Graphite is different from other metals in that it is a layered material. Each layer consists of hexagonal rings formed by $\mathrm{sp}^{2}$ hybridized orbitals of carbon atoms. The remaining unhybridized p orbitals point normal to the layers and form $\pi$ bonds. The $\pi$ electrons are what give rise to the semimetallic properties of graphite and its large anisotropy of electrical conductivity. For example, the ratio of the conductivity in a layer to that between layers can be as high as $10^{5} .{ }^{41}$ The interlayer distance of 3.35 $\AA$ is almost 2.5 times the nearest neighbor separation of $1.14 \AA$ within a layer. The large interlayer separation makes the exact location of an image plane inside HOPG unclear. Thus, choosing a perfect image charge for TOPO inside HOPG in our calculations is questionable. From another perspective, the screening length for HOPG is $8.5 \AA .{ }^{42}$ Classical image charge theory can only be applied when the external charge is at a distance $z$ from the metal, where $z$ is greater than the screening length of the metal. When the charge approaches the surface to within the screening length of the metal, an exchangecorrelation hole is formed. ${ }^{43}$ Thus, the classical image charge becomes a diffused electron cloud. ${ }^{43}$ Therefore, the effective image charge would be equivalent to a partial image charge.
Due to the low density of states near the Fermi level, when a voltage was placed across HOPG, it was found that a considerable potential drop inside the graphite occurred. ${ }^{42,44}$ This is not the case for a perfect metal. Thus, it is possible that any applied voltage is not completely dropped across the tip and HOPG surface. This would lead to an error in measured $V_{\mathrm{dc}}+$ $\phi$. From the magnitude of the potential drop in HOPG, the space charge capacitance was calculated to have a minimum value of $3.42 \mu \mathrm{F} / \mathrm{cm}^{2}$ at zero applied voltage. ${ }^{42,44}$ This value is one or 2 orders of magnitude bigger than the tip-substrate capacitance in our EFM measurement. To model the effects of the space charge capacitance on our data, we can treat the tip-graphite capacitance as two capacitors in series. One is the tip-substrate surface capacitor, and the other is a space charge capacitor inside the HOPG. Since the tip-substrate surface capacitance is so small relative to the space charge capacitance, the voltage drop inside graphite is negligible in our experiments.

The magnitude of the force gradient over a TOPO stripe differs between the experiments and calculations by a factor of 6.5. This difference could possibly be due to the inaccuracy of the value of $V_{\mathrm{dc}}+\phi=0.02 \mathrm{~V}$. A value of $0.02 \mathrm{~V}$ is very small compared to the contact potential difference $\phi \sim 0.5 \mathrm{~V}$. Also, $\phi$ is a local property, and it varies over the graphite surface by $\sim 15 \mathrm{mV}$. Furthermore, the uncertainty in the cantilever force constant of a factor of $\sim 3$ introduces another source of possible error.

From the contact potential difference versus $z$ measurements shown in Figure 4, we conclude that graphite does not behave like gold, a good metal. For a metal $\phi$ should not be a function of the separation between the surface and the tip. The origin of the anomalous behavior of HOPG is unclear. One possible 
explanation concerns the intrinsic electrostatic properties of a graphite surface. Some studies claim that there exists a quadrupole field in the basal plane of the graphite due to the similarity between graphite and aromatic compounds such as benzene, which all have a quadrupole moment. ${ }^{45}$ An electric field that has $z$ dependence, such as a quadrupole field, would produce the same change in $\phi(z)$ as what we observe.

\section{Conclusion}

We have studied the behavior of TOPO self-assembly on graphite using AFM. We observe the formation of a crystalline stripe structure in registry with the underlying graphite lattice. The EFM results show that the adsorbed TOPO layer is positively charged, with $\sim 2 \times 10^{-5}$ electron charge per TOPO molecule if we take the area of one TOPO molecule in the stripe to be $1 \mathrm{~nm}^{2}$. The TOPO dipole is nearly parallel to the surface yielding a small dipole EFM signal. The EFM data show that HOPG does not form perfect image charges, apparently because of the large interlayer separation and the low density of states near the Fermi level.

Detailed knowledge of TOPO self-assembly on HOPG, such as the molecular orientation and the adsorption site on HOPG, is very limited. Also, image charge formation in HOPG is not well explored. It would be interesting to investigate a wellcharacterized system, such as alkanethiols on gold. Alkanethiol SAMs on gold have been studied extensively, ${ }^{4,11}$ and gold is also a perfect metal. By using techniques such as "dip-pen" nanolithography 46 to "write" arbitrary-shaped alkanethiol structures on gold, we could gain a better understanding about the electric field of dipole layers on metal surfaces.

Acknowledgment. We thank the support of the W. M. Keck Foundation in the purchase of the AFM and Bell Laboratories for equipment donation. This research was supported under NSF MRSEC Grant No. DMR-98-09687 for materials research at Columbia University.

\section{References and Notes}

(1) Swalen, J. D.; Allara, D. L.; Andrade, J. D.; Chandross, E. A.; Garoff, S.; Israelachvili, J.; McCarthy, T. J.; Murray, R.; Pease, R. F.; Rabolt, J. F.; Wynne, K. J.; Yu, H. Langmuir 1987, 3, 932.

(2) Collier, C. P.; Wong, E. W.; Belohradský, M.; Raymo, F. M.; Stoddart, J. F.; Kuekes, P. J.; Williams, R. S.; Heath, J. R. Science 1999, $285,391$.

(3) Ulman, A. Chem. Rev. 1996, 96, 1533.

(4) Bain, C. D.; Troughton, E. B.; Tao, Y.; Evall, J.; Whitesides, G. M.; Nuzzo, R. G. J. Am. Chem. Soc. 1989, 111, 321.

(5) Gorman, C. B.; Touzov, I.; Miller, R. Langmuir 1998, 14, 3052.

(6) Martin, B.; Ockenden, D. W.; Foreman, J. K. J. Inorg. Nucl. Chem. 1961, 21, 96.
(7) Cecconie, T.; Freiser, H. Anal. Chem. 1990, 62, 622.

(8) Murray, C. B.; Norris, D. J.; Bawendi, M. G. J. Am. Chem. Soc. 1993, 115,8706 .

(9) Alivisatos, A. P. J. Phys. Chem. 1996, 100, 13226.

(10) Collier, C. P.; Vossmeyer, T.; Heath, J. R. Annu. Rev. Phys. Chem. 1998, 49, 371 .

(11) Fenter, P.; Eberhardt, A.; Eisenberger, P. Science 1994, 266, 1216.

(12) Binning, G.; Rohrer, H.; Gerber, Ch.; Weibel, E. Phy. Rev. Lett. 1982, 49, 57.

(13) Binnig, G.; Quate, C. F.; Gerber, Ch. Phys. Rev. Lett. 1986, 56, 930.

(14) Widrig, C. A.; Alves, C. A.; Porter, M. D. J. Am. Chem. Soc. 1991, $113,2805$.

(15) Poirier, G. E.; Pylant, E. D. Science 1996, 272, 1145.

(16) Martin, Y.; Abraham, D. W.; Wickramasinghe, H. K. Appl. Phys. Lett. 1988, 52, 1103

(17) Stern, J. E.; Terris, B. D.; Mamin, H. J.; Rugar, D. Appl. Phys. Lett. 1988, 53, 2717.

(18) Terris, B. D.; Stern, J. E.; Rugar, D.; Mamin, H. J. Phys. Rev. Lett. 1989, 63, 2669.

(19) Krauss, T. D.; Brus, L. E. Phys. Rev. Lett. 1999, 83, 4840.

(20) Schönenberger, C.; Alvarado, S. F. Phys. Rev. Lett. 1990, 65, 3162.

(21) Nonnenmacher, M.; O'Boyle, M. P.; Wickramasinghe, H. K. Appl. Phys. Lett. 1991, 58, 2921.

(22) Böhmisch, M.; Burmeister, F.; Rettenberger, A.; Zimmermann, J.; Boneberg, J.; Leiderer, P. J. Phys. Chem. B 1997, 101, 10162.

(23) Weaver, J. M. R.; Abraham, D. W. J. Vac. Sci. Technol. B 1991, 9, 1559.

(24) Saurenbach, F.; Terris, B. D. Appl. Phys. Lett. 1990, 56, 1703.

(25) Bergasa, F.; Sáenz, J. J. Ultramicroscopy 1992, 42-44, 1189.

(26) Martin, Y.; Williams, C. C.; Wickramasinghe, H. K. J. Appl. Phys. 1987, 61, 4723 .

(27) Evans, S. D.; Ulman, A. Chem. Phys. Lett. 1990, 170, 462.

(28) Annett, J. F.; Palmer, R. E.; Willis, R. F. Phys. Rev. B 1988, 37, 2408.

(29) Penner, R. M. Acc. Chem. Res. 2000, 33, 78.

(30) ZYB grade, Advanced Ceramics Corp.

(31) TESP and MESP cantilevers (Digital Instruments).

(32) Lorenz, J. K.; Ellis, A. B. J. Am. Chem. Soc. 1998, 120, 10970

(33) Belaidi, S.; Girard, P.; Leveque, G. J. Appl. Phys. 1997, 81, 1023.

(34) Borovikov, Yu. Ya.; Ryl'tsev, E. V.; Boldeskul, I. E.; Feshchenko,

N. G.; Makovetskii, Yu. P.; Egorov, Yu. P. J. Gen. Chem. U.S.S.R. 1970, 40, 1942.

(35) Kardar, M.; Berker, A. N. Phys. Rev. Lett. 1982, 48, 1552.

(36) Uphaus, R. A.; Vandegrift, G. F.; Horwitz, E. P. J. Colloid Interface Sci. 1982, 90, 380 .

(37) Anastopoulos, A.; Karabinas, P.; Jannakoudakis, D. J. Electroanal. Chem. 1981, 127, 219.

(38) Rabe, J. P.; Buchholz, S. Science 1991, 253, 424.

(39) Groszek, A. J. Proc. R. Soc. London A 1970, 314, 473.

(40) Hammond, W. R.; Mahanti, S. D. Surf. Sci. 1990, 234, 308.

(41) Brandt, N. B.; Chudinov, S. M.; Ponomarev, Ya. G. Semimetals, Graphite and its Compounds. Modern Problems in Condensed Matter Sciences; North-Holland: Amsterdam, 1988; Vol. 20.1.

(42) Gerischer, H. J. Phys. Chem. 1985, 89, 4249.

(43) Sutton, A. P. Electronic Structure of Materials; Oxford University Press Inc.: New York, 1993.

(44) Gerischer, H.; McIntyre, R.; Scherson, D.; Storck, W. J. Phys. Chem. 1987, 91, 1930.

(45) Vernov, A.; Steele, W. A. Langmuir 1992, 8, 155.

(46) Piner, R. D.; Zhu, J.; Xu, F.; Hong, S.; Mirkin, C. A. Science 1999, 283,661 . 